\title{
Study of the role of the MtWOX9-1 gene in somatic embryogenesis
}

\author{
Baltin S.M.*, Tvorogova V.E., Lutova L.A. \\ St. Petersburg State University, St. Petersburg, Russia \\ *email: krubaza@mail.ru
}

Somatic embryogenesis (SE) in plants is the process by which non-zygotic cells form embryos, which go through characteristic stages of embryonic development, ultimately forming a new plant. Many plant species are capable of SE, but in many of them, the formation of somatic embryos requires specific in vitro conditions. SE is widely used for the genetic transformation of plants and for the production of artificial seeds, and the study of its regulators is necessary to improve the methods of obtaining somatic embryos. SE has many similarities with zygotic embryogenesis (ZE): during the somatic embryo development, morphological stages characteristic of ZE can usually be distinguished; moreover, most of the studied $\mathrm{ZE}$ regulators function also during SE. Transcription factors from the WUSCHEL-LIKE HOMEOBOX family (WOX2, WOX8 and WOX9 and others) can be referred to as ZE regulators. Previously in our research, a role of some of their orthologs in the SE in Medicago truncatula was revealed. For example, overexpression of MtWOX9-1 increases SE capacity and changes the level of transcription of many genes. The aim of our study is to further investigate the role of the MtWOX9-1 genes. The main goals include investigating the inactivation of the Medicago truncatula MtWOX9-1 gene using the CRISPR/Cas9 system. At this moment, plants carrying alleles with loss of MtWOX9-1 function have been obtained, and in the future we plan to assess the SE capacity of their offspring.

Acknowledgments: The research was made with support of the Ministry of Science and Higher Education of the Russian Federation in accordance with agreement No. 075-152020-922 date 16.11.2020 on providing a grant in the form of subsidies from the Federal budget of Russian Federation. The grant was provided for state support for the creation and development of a World-class Scientific Center "Agrotechnologies for the Future". 